Research article

\title{
Weekly intra-articular injections of bone morphogenetic protein-7 inhibits osteoarthritis progression
}

\author{
Masaya Hayashi ${ }^{1}$, Takeshi Muneta ${ }^{1}$, Young-Jin Ju${ }^{1}$, Tomoyuki Mochizuki ${ }^{2}$ and Ichiro Sekiya ${ }^{2}$ \\ ${ }^{1}$ Section of Orthopaedic Surgery, Graduate School, Tokyo Medical and Dental University, Yushima, Bunkyo-ku, Tokyo, $113-8519$ Japan \\ ${ }^{2}$ Section of Cartilage Regeneration, Graduate School, Tokyo Medical and Dental University, Yushima, Bunkyo-ku, Tokyo, $113-8519$ Japan \\ Corresponding author: Ichiro Sekiya, sekiya.orj@tmd.ac.jp
}

Received: 28 Nov 2007 Revisions requested: 18 Jan 2008 Revisions received: 4 Sep 2008 Accepted: 30 Sep 2008 Published: 30 Sep 2008

Arthritis Research \& Therapy 2008, 10:R118 (doi:10.1186/ar2521)

This article is online at: http://arthritis-research.com/content/10/5/R118

(C) 2008 Hayashi et al.; licensee BioMed Central Ltd.

This is an open access article distributed under the terms of the Creative Commons Attribution License (http://creativecommons.org/licenses/by/2.0), which permits unrestricted use, distribution, and reproduction in any medium, provided the original work is properly cited.

\begin{abstract}
Introduction We investigated the ability of a weekly intraarticular injection of bone morphogenetic protein (BMP)-7 to prevent osteoarthritis in rabbits with anterior cruciate ligament transections.

Methods First, 36 knee joints were randomly divided into four groups: 50, 500, 5,000 ng BMP-7, and control. Knee cartilage was evaluated at 4,8 , and 12 weeks. Then, in order to control for individual differences, $500 \mathrm{ng} \mathrm{BMP-7}$ was injected into one knee and phosphate-buffered saline (PBS) into the other, and the two knees were compared at 4,8 , and 12 weeks $(n=5)$. For pharmacokinetic analysis, cartilage was harvested at 1 hour and $1,2,4$, and 7 days after knee injection of $500 \mathrm{ng}$ BMP-7 or PBS $(n=3)$.
\end{abstract}

Results Histological scores in the 500 and 5,000 ng BMP-7 groups were significantly better than those in the other groups at 12 weeks. Matched pair analysis demonstrated that both macroscopic and histological scores in the $500 \mathrm{ng}$ BMP-7 group were better than those in the control group. Immunohistochemical analysis revealed higher BMP-7 expression by chondrocytes in the BMP-7 injected knees. Histology of whole knee and quantitative micro computed tomography analysis showed that weekly injections of $500 \mathrm{ng}$ BMP-7 did not induce synovial fibrosis, ectopic bone, or osteophyte formation. As detected by enzyme-linked immunosorbent assay, BMP-7 concentration in the cartilage tissue was still higher in the BMP-7 treated group 7 days after the injection.

Conclusions Weekly intra-articular injections of BMP-7 inhibited progression of osteoarthritis. Obvious adverse effects were not observed. BMP-7 concentration and expression in cartilage were still higher 7 days after injection.

\section{Introduction}

Osteoarthritis of the knee is one of the leading causes of disability among elderly people. It is mainly caused by the breakdown and eventual loss of joint cartilage. For many years, scientists have been searching for ways to intervene in the disease process and so retard or even prevent progression of joint damage.

Bone morphogenetic protein (BMP)-7, also referred to as osteogenic protein-1, has a profound effect on chondrocyte metabolism by stimulating the synthesis [1-3], organization [4], and retention [2,5] of matrix molecules. With increasing age and progression of articular cartilage degeneration, the expression level of endogenous BMP-7 decreases [6], sug- gesting that a decrease in BMP-7 may play an important role in the progression of cartilage degeneration. A recent study [7] demonstrated that continuous intra-articular infusion of BMP-7 had a protective effect on cartilage degeneration, which suggests the possible utility of BMP-7 as a treatment for human osteoarthritis.

We speculated that periodic knee injections of a small amount of BMP-7 would suppress the loss of cartilage matrix and consequently prevent osteoarthritis progression, without any adverse drug effects. In this study, BMP-7 was injected weekly into the knee joints of rabbits after anterior cruciate ligament transection (ACLT), and the cartilage of the knee was evaluated morphologically. The knees were also evaluated for any 
possible adverse effects of the BMP-7. Furthermore, BMP-7 concentration in cartilage was sequentially analyzed after intraarticular injection of BMP-7. The overall results suggest that weekly intra-articular injection of a small amount of BMP-7 is a promising nonsurgical treatment for osteoarthritis, and that BMP-7 is an interesting candidate structure/disease-modifying osteoarthritis drug.

\section{Materials and methods}

\section{Animals and injection of BMP-7}

Skeletally mature female Japanese white rabbits $(10 \pm 2$ months old) weighing an average of $3.2 \mathrm{~kg}$ (range 2.8 to 3.6 $\mathrm{kg}$ ) were used in the experiments. This study was conducted in accordance with a protocol approved by the Animal Committee of Tokyo Medical and Dental University. Animals underwent bilateral ACLT under anesthesia induced by intramuscular injection of $25 \mathrm{mg} / \mathrm{kg}$ ketamine hydrochloride (Sankyo, Tokyo, Japan) and intravenous injection of $45 \mathrm{mg} / \mathrm{kg}$ sodium pentobarbital (Dainippon Sumitomo Pharma, Osaka, Japan). The knee joint was approached through a medial parapatellar incision, and the patella was dislocated laterally. The anterior cruciate ligament was then transected with a sharp blade, and the capsule was sutured to render it watertight, followed by skin closure. All animals were allowed normal cage activity.

Lyophilized 5\% lactose-buffered recombinant human BMP-7 (rhBMP-7; Stryker Biotech, Hopkinton, MA, USA) was dissolved in phosphate-buffered saline (PBS). Aliquoted 50, 500, or 5,000 ng BMP-7 in $200 \mu \mathrm{l}$ PBS was administrated intraarticularly with a 27 -gauge needle on a $1.0 \mathrm{ml}$ syringe through the lateral infrapatellar area toward the intercondylar space of the femur in each animal in a deep knee-flexed position. The first injection was given immediately after ACLT; the second and subsequent injections were administered once a week up to 12 weeks. The final injection was administered a week before the animals were killed, by an overdose of sodium pentobarbital; the knee joints were then evaluated.

For evaluation of the optimal dose of BMP-7, 18 rabbits with 36 knees were used. All animals underwent bilateral ACLT. The 36 knee joints were randomly assigned to one of four groups: three doses $(50,500$, or $5,000 \mathrm{ng})$ of BMP-7 or control (PBS alone).

For matched pair analyses of BMP-7, 15 rabbits with 30 knees were used. The other three rabbits with six knees were used for whole knee experiments conducted to investigate the intraarticular influences of BMP-7. The dose of $500 \mathrm{ng}$ was chosen based on the previous results. In each of the 18 animals, BMP7 was injected into the right knee and PBS into the left, as a control.

\section{Gross morphological examination}

Femoral condyles were dissected and stained with India ink. Macroscopic pictures were taken using MPS-7 (Sugiura Laboratory Inc., Tokyo, Japan), a dedicated medical photography platform, and used for macroscopic evaluation. Digital images were taken using a Nikon Coolpix 4500 digital camera (Nikon, Tokyo, Japan). Gross findings were classified into six grades (grade 1: intact articular surface; grade 2: minimal fibrillation; grade 3: overt fibrillation; grade 4a: erosion of 0 to $2 \mathrm{~mm}$; grade $4 \mathrm{~b}$ : erosion of 2 to $5 \mathrm{~mm}$; and grade $4 \mathrm{c}$ : erosion of $>5$ $\mathrm{mm}$ ) and scored accordingly [8]. Both the medial and lateral femoral condyles were individually scored. Then, the two scores were summed to obtain a cumulative macroscopic osteoarthritis score. In a blinded manner, the assessment was conducted by two independent examiners, who were blinded to each other's findings and to the treatment group assignment of the animals. Finally, the two scores from the examiners were averaged to obtain an overall score.

\section{Histological examination}

The dissected distal femurs were fixed in a 4\% paraformaldehyde solution after gross morphological examination. The specimens were decalcified in 4\% EDTA solution, dehydrated with a gradient ethanol series, and embedded in paraffin blocks. Based on macroscopic observation, 20 coronal sections per knee were carefully prepared so as to include the most severely degenerated area. For whole knee specimens, sagittal sections were stained with Masson's trichrome. Histological sections were visualized using an Olympus IX71 microscope (Olympus, Tokyo, Japan) and PIXERA Viewfinder 3.0 software (Pixera Corporation, San Jose, CA, USA). Histological sections were assessed in a blinded manner by two individual examiners, who were unaware of the treatment group assignment of the animals, and quantified using the advanced grading methodology of the Osteoarthritis Research Society International (OARSI) osteoarthritic cartilage histopathology grading system [9].

\section{Immunohistochemical analysis}

Paraffin-embedded sections were deparaffinized in xylene, rehydrated through graded alcohol, and immersed in PBS. The samples were pretreated with $0.4 \mathrm{mg} / \mathrm{ml}$ proteinase $\mathrm{K}$ (DAKO, Carpinteria, CA, USA) in Tris- $\mathrm{HCl}$ buffer for 15 minutes at room temperature for antigen retrieval. Any residual enzymatic activity was removed by washing with PBS, and nonspecific staining was blocked by preincubation with PBS containing $10 \%$ normal horse serum for 20 minutes at room temperature. Mouse monoclonal anti-BMP-7 antibody (12G3; 1:100 dilution; Stryker Biotech, Hopkinton, MA, USA) was placed on the sections overnight at $4^{\circ} \mathrm{C}$. After extensive washing with PBS, the sections were incubated in the secondary antibody of biotinylated horse anti-mouse IgG (Vector Laboratories, Burlingame, CA, USA) for 30 minutes at room temperature. Immunostaining was detected with VECTASTAIN ABC reagent 
(Vector Laboratories), followed by DAB staining. Counterstaining was performed with methyl green.

\section{Semi-quantitative analysis of synovial fibrosis}

The whole knee specimens stained with Masson's trichrome were analyzed in order to measure the rate of synovial fibrosis in the infrapatellar fat pad (IPF) $[10,11]$. The area of the whole IPF and blue stained area of collagen fibers was measured using Scion Image software (Scion Corporation, Frederick, MD, USA). The rate of fibrosis in IPF (\% fibrosis) was calculated as the blue stained area divided by the whole IPF area $x$ 100.

\section{Micro computed tomography scanning and quantification of osteophyte volume}

All specimens were subjected to analysis using a high-resolution micro computed tomography scanner (ScanXmate-E090; Comscantecno, Kanagawa, Japan). Osteophytes were manually traced and detected in 10 axial sections, interpolated, reconstituted, and quantified using TRI/3D-BON software (RATOC, Tokyo, Japan).

\section{Pharmacokinetic analysis of BMP-7 in cartilage tissue}

At 1 hour and 1, 2, 4, and 7 days after intra-articular injection of 500 ng BMP-7 or PBS into normal knees, rabbits were killed, and cartilage tissue from their knee joints was collected. The cartilage was homogenized in CelLytic ${ }^{\mathrm{TM}}$ MT Mammalian Tissue Lysis/Extraction Reagent (Sigma, St. Louis, MO, USA) and the lysed samples were centrifuged for 10 minutes at $12,000 \mathrm{~g}$. The supernatant was stored with protease inhibitor cocktail (Sigma, St. Louis, MO, USA) and BMP-7 levels were measured using a sandwich enzyme-linked immunosorbent assay. Monoclonal 1B12 antibody (Stryker Biotech, Hopkinton, MA, USA) was utilized as a coating antibody. Plates were coated with $1 \mu \mathrm{g} /$ well of this antibody in sodium carbonatebicarbonate buffer and incubated overnight at $4^{\circ} \mathrm{C}$. Nonspecific binding was blocked with $4 \%$ milk/borate-buffered saline containing $0.05 \%$ Tween-20 (BBST) blocking buffer. Either BMP-7 standard or cartilage extract was added to the plate and incubated at $37^{\circ} \mathrm{C}$ for 1 hour. A second anti-BMP-7 antibody labeled with alkaline phosphatase (12G3-AP) was applied at 1:100 dilution in sodium carbonate-bicarbonate buffer and incubated at $37^{\circ} \mathrm{C}$ for 1 hour. After this incubation, an alkaline phosphatase substrate, PNPP-phosphatase substrate (Pierce, Rockford, IL, USA), was added and incubated at room temperature for 30 minutes. To stop the reaction, $2 \mathrm{~N}$ $\mathrm{NaOH}$ was added. The absorbance was detected and quantitated by microplate reader Sunrise Remote (TECAN, Männedorf, Switzerland). The data were then processed in LSPLATEmanager software (Wako, Osaka, Japan).

\section{Statistical analysis}

All data are expressed as mean \pm standard deviation. A nonparametric Mann-Whitney $U$ test was used to evaluate the statistical significance of differences in the macroscopic and histologic results. A Wilcoxon's signed rank-sum test was used to perform matched pair analyses. Interobserver variation in gross morphologic grading was verified by measuring agreement with the $\kappa$ statistic. $P$ values less than 0.05 were considered statistically significant.

\section{Results \\ Dose effect of BMP-7 for preventing the progression of osteoarthritis}

After ACLT, 50, 500, or 5,000 ng BMP-7 in $200 \mu \mathrm{l}$ PBS or PBS alone was injected weekly into the knee joint. Macroscopic observation of femoral condyles at 12 weeks demonstrated obvious surface irregularity in the control and $50 \mathrm{ng}$ BMP-7 groups, whereas a milder alteration in the articular surface was observed in the $500 \mathrm{ng}$ and 5,000 ng BMP-7 groups (Figure 1a). No damage was caused by the needle. Histologically, cartilage matrix disappeared in the control and $50 \mathrm{ng}$ BMP-7 groups, whereas it was predominantly retained in the $500 \mathrm{ng}$ and 5,000 ng BMP-7 groups (Figure 1b). The OARSI osteoarthritis scores for histologic analysis were similar in all four groups at 4 and 8 weeks; however, they were significantly better in the $500 \mathrm{ng}$ and $5,000 \mathrm{ng}$ BMP-7 groups than in the control group at 12 weeks (Figure 1c). These data indicate that weekly injections of $500 \mathrm{ng}$ and 5,000 ng BMP-7 prevented the progression of osteoarthritis. There were no significant differences between the $500 \mathrm{ng}$ and 5,000 ng groups. The dose-response effect of BMP-7 reached a plateau at 500 $\mathrm{ng}$, and therefore the dose of $500 \mathrm{ng}$ BMP-7 was used in further analyses.

\section{Matched pair analyses: effect of BMP-7 on osteoarthritis progression}

During the early stage of the investigation, we found that rabbits exhibited considerable individual variability in osteoarthritis progression after ACLT. To examine the effect of BMP-7 on osteoarthritis progression in a stricter manner, after the ligaments in the both knees were dissected, 500 ng BMP-7 in $200 \mu$ PBS was injected into the right knee, and the same amount of PBS was injected into the left knee of the same animal on a weekly basis.

Macroscopic observations on gross morphologic changes of the femoral condyles in the control group revealed subtle cartilage lesions at 4 weeks, a slight progression at 8 weeks, and obvious surface irregularity at 12 weeks (Figure 2a). On the contrary, cartilage of the femoral condyles in the treatment group appeared to be better throughout the study. Interestingly, four out of five rabbits in the control side/group exhibited erosions in both the lateral and medial femoral condyles at 12 weeks; however, in the 500 ng BMP-7 side/group, only two rabbits exhibited erosions in the lateral femoral condyle, and two other rabbits showed erosions in the medial femoral condyle. In all animals, the macroscopic osteoarthritis score was better in the BMP-7 injected knee than in the contralateral control knee (Figure 2b). The interobserver agreement for 
(a)

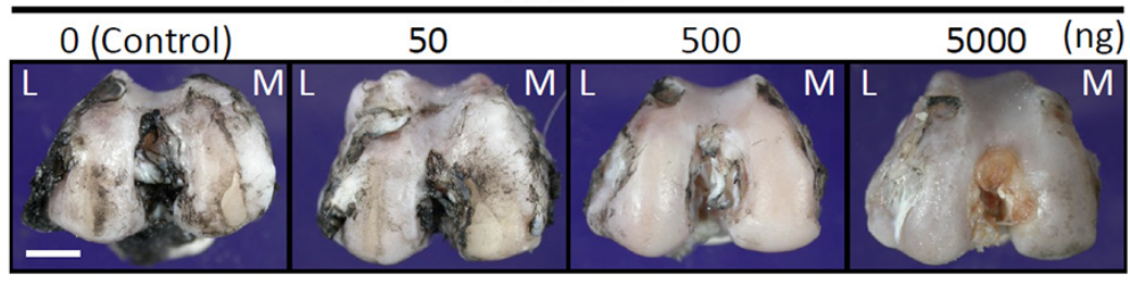

(b)

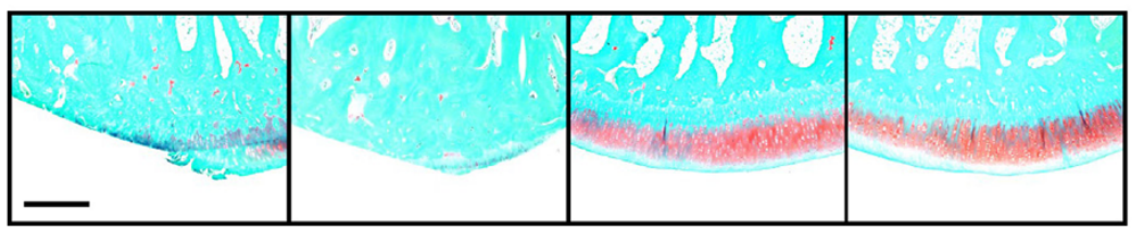

(c)

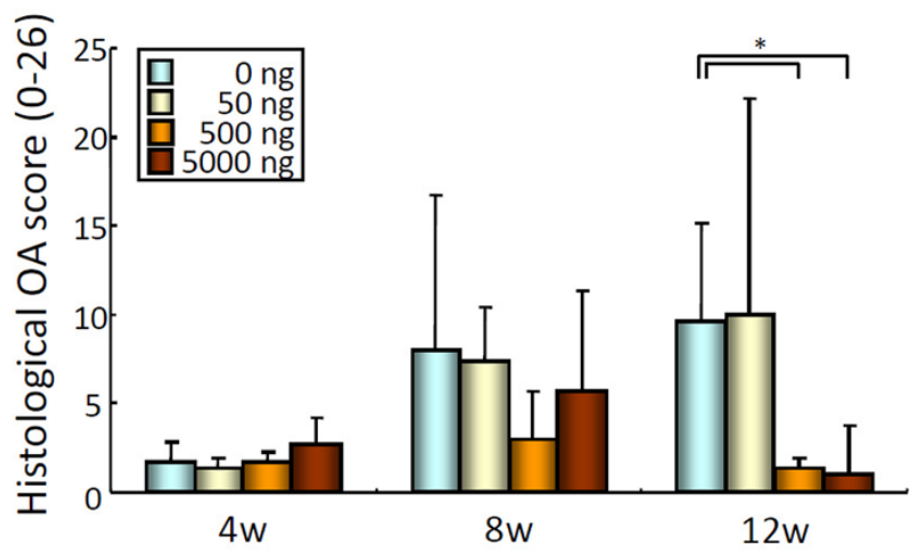

Evaluation of dose effect of BMP-7 for prevention of osteoarthritis progression in rabbit ACLT. (a) Representative macroscopic appearances of the distal portion of the femoral condyles at 12 weeks. Surface of the cartilage was stained with India ink to identify any fibrillation and erosion. Laterality is shown as lateral (L) and medial (M). Bar $=5 \mathrm{~mm}$. (b) Representative histology of femoral condyles at 12 weeks. Distal femur was sectioned coronally and stained with safranin-O. The most degenerated area of each sample is included. Bar $=50 \mu \mathrm{m}$. (c) Quantitation of histological analysis using the OARSI cartilage osteoarthritis histopathology grading system. The scores are displayed as average \pm standard deviation (three knees). ${ }^{*} P$ $<0.05$, by Mann-Whitney U test. ACTL, anterior cruciate ligament transection; BMP, bone morphogenetic protein; OARSI, Osteoarthritis Research Society International.

grading of cartilage damage indicates high reproducibility $(\kappa=$ 0.84).

Histologically, stainability with safranin- $O$ in cartilage matrices appeared to be similar in both the $500 \mathrm{ng} \mathrm{BMP-7}$ and control groups at 4 weeks (Figure 2c). At 8 weeks, in the control side/ group, cartilage became thinner or stainability with safranin-O became worse than in the BMP-7 side/group. At 12 weeks, cartilage lesions further worsened, and most of the knees in the control side/group exhibited severe erosion or cartilage defects in both the medial and lateral condyles. BMP-7 injected knees also developed erosive lesions; however, those were limited either to the medial or to the lateral femoral condyle. The OARSI osteoarthritis score was better at the BMP-7 side than at the contralateral control side in each rabbit at 12 weeks (Figure 2d). Matched pair analyses revealed that weekly 500 ng BMP-7 injections slowed the progression of osteoarthritis.

\section{BMP-7 expression in cartilage of knee after weekly BMP- 7 injection}

Immunohistochemical analysis showed that BMP-7 was barely expressed in chondrocytes of the remaining cartilage in the control knees. On the contrary, the rate of chondrocytes staining positive for BMP-7 was higher in the knees receiving treatment with BMP-7 despite 7 days having passed since the last injection (Figure 3).

\section{Investigation of presumable adverse effects of BMP-7}

It was hypothesized that repeated BMP-7 injections into the knee joint might induce adverse effects such as synovial fibrosis, ectopic cartilage and bone formation, or osteophyte forma- 

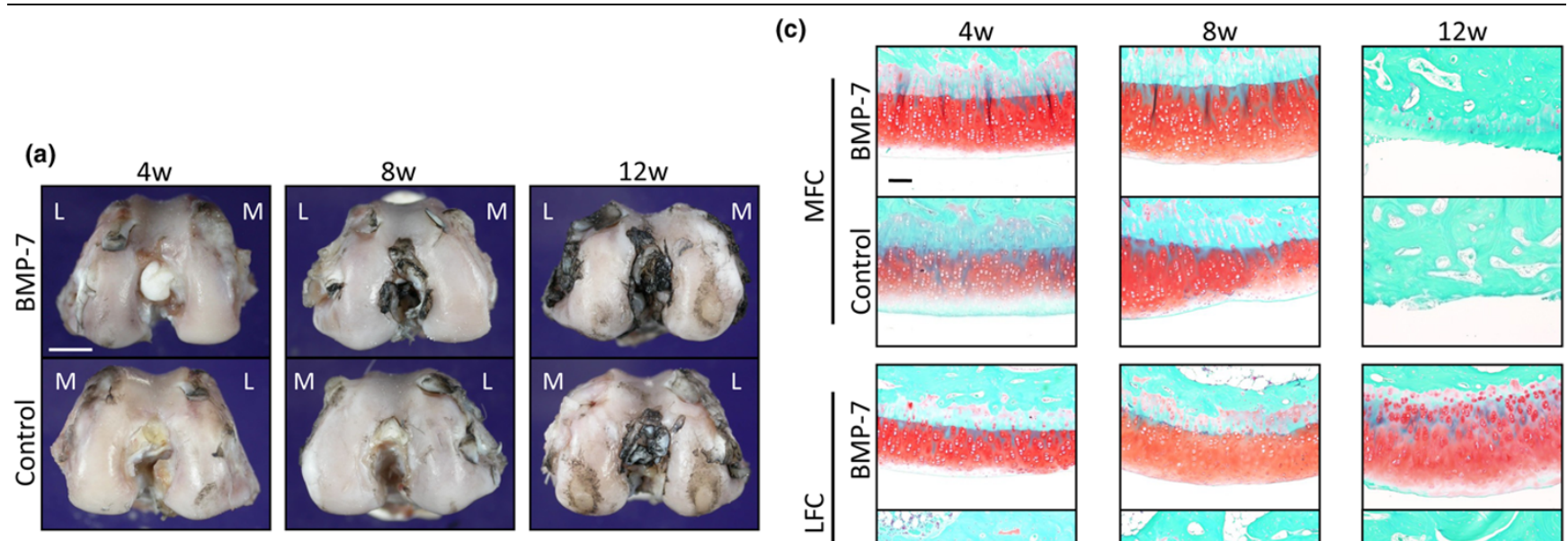

(b)
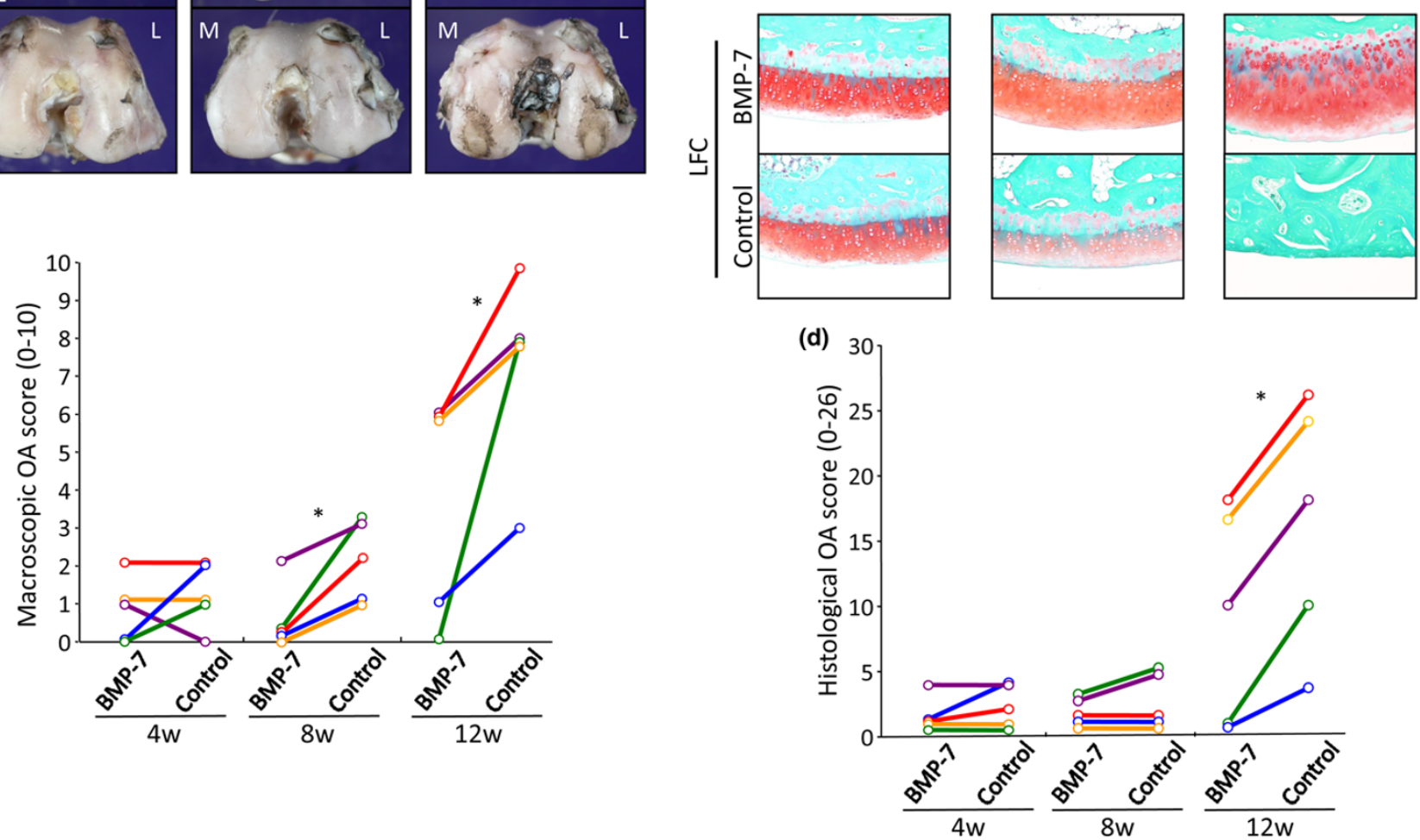

Matched pair analyses of the effect of BMP-7 on osteoarthritis progression. (a) Macroscopic appearances of the femoral condyles at 4, 8, and 12 weeks. To remove individual variability, both sides of the knees of the same individuals are shown. Surface of the cartilage was stained with India ink to identify any fibrillation and erosion. Laterality is shown as lateral $(\mathrm{L})$ and medial $(\mathrm{M})$. Bar $=5 \mathrm{~mm}$. (b) Paired comparison in the macroscopic osteoarthritis score. The scores of the treatment and control knees (in the same animal) are displayed separately and connected with a line (five knees). ${ }^{\star} P<0.05$, by Wilcoxon's signed rank sum test. (c) Representative histology of femoral condyles. Both sides of the knees from the same individuals are shown. Medial femoral condyles (MFC) and lateral femoral condyles (LFC) were sectioned coronally and stained with safranin-O. Bar $=10 \mu \mathrm{m}$. (d) Paired comparison in quantitation of histological analysis using the OARSI cartilage osteoarthritis histopathology grading system (five knees). ${ }^{*} P$ $<0.05$ by Wilcoxon's signed rank sum test for paired samples. BMP, bone morphogenetic protein; OARSI, Osteoarthritis Research Society International.

tion. Various techniques were used to screen for any possible unwanted response.

For synovial fibrosis, fibrotic areas in the IPF were compared. According to the quantitative analysis, the ratio of fibrotic area in the BMP-7 treated knee was not higher than that in the control knee (Figure 4). We examined the area around the border between cartilage and synovium, the area around the sutured capsule, and the area around the dissected anterior cruciate ligament carefully, but we did not detect ectopic cartilage formation in the knees injected with BMP-7. For ectopic bone or osteophyte formation, whole knee joints were evaluated by micro computed tomography. No ectopic bone formation was observed by three-dimensional reconstructed imaging (Figure 5a). Quantitative analysis showed that the volume of osteophyte formation in the BMP-7 group was not more than that in the control group at 12 weeks (Figure 5b,c). Based on these results, it appears that BMP-7 did not induce any obvious adverse effects.

\section{Pharmacokinetic analysis of BMP-7 in cartilage tissue}

BMP-7 levels in cartilage tissue were measured using sandwich enzyme-linked immunosorbent assay (Figure 6a). In control knees, BMP-7 was barely detected at any of the 
Figure 3

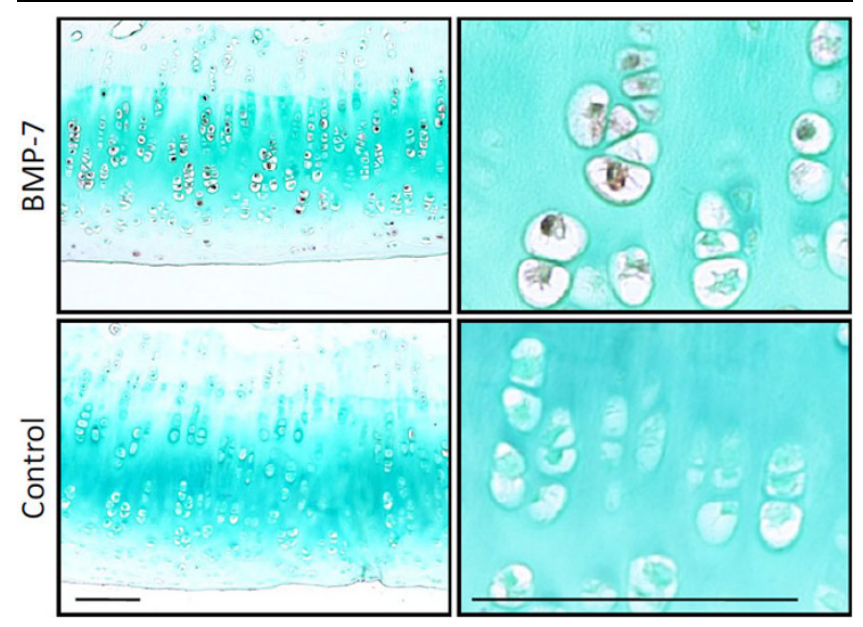

Immunohistochemical analysis for BMP-7 in cartilage. After anterior cruciate ligament transection in both knees, $500 \mathrm{ng}$ BMP-7 in $200 \mu \mathrm{l}$ PBS was injected weekly into the right knee and the same amount of PBS was injected into the left knee. The remaining cartilage in medial femoral condyles was evaluated at 12 weeks ( 7 days after the last injection). Higher magnifications are demonstrated in the right column. Bars $=10$ $\mu \mathrm{m}$. BMP, bone morphogenetic protein; PBS, phosphate-buffered saline.

observation times (Figure 6b). On the contrary, 1 hour after BMP-7 injection BMP-7 was detected at high levels in cartilage; the level decreased by $60 \% 1$ day after injection. BMP7 levels gradually decreased over time, and 7 days after the last injection BMP-7 concentration in treated knees was still higher than that in control knees.

\section{Discussion}

Experimental osteoarthritis induced by ACLT is one of the most widely used models [12-14] and provides temporal progression of cartilage degeneration in the knee. One of the characteristics of this model is that cartilage is affected initially from medial femoral condyle and eventually extends over the lateral femoral condyle [15]. This model alters both the magnitude and distribution of joint forces that are applied to the cartilage surface in vivo. Joint instability is the key factor for the onset and/or progression of cartilage degeneration in human osteoarthritis, and this model mimics that mechanism. Because of the instability generated, it is a particularly challenging model for testing structure/disease-modifying osteoarthritis drugs, because the instability that caused the osteoarthritis is still present even as the drug is being applied.

In animal cartilage defect models, several reports have shown that implantation of a scaffold impregnated or mixed with BMP-2 [16] or BMP-7 [17-21] can promote cartilage repair. However, it is expected that a single administration of BMP will not prevent progression of osteoarthritis induced by joint instability. To overcome this potential problem, an osmotic pump was previously used [7]; however, from the standpoint of clinical availability, periodic injections into the knee of BMP-7
Figure 4
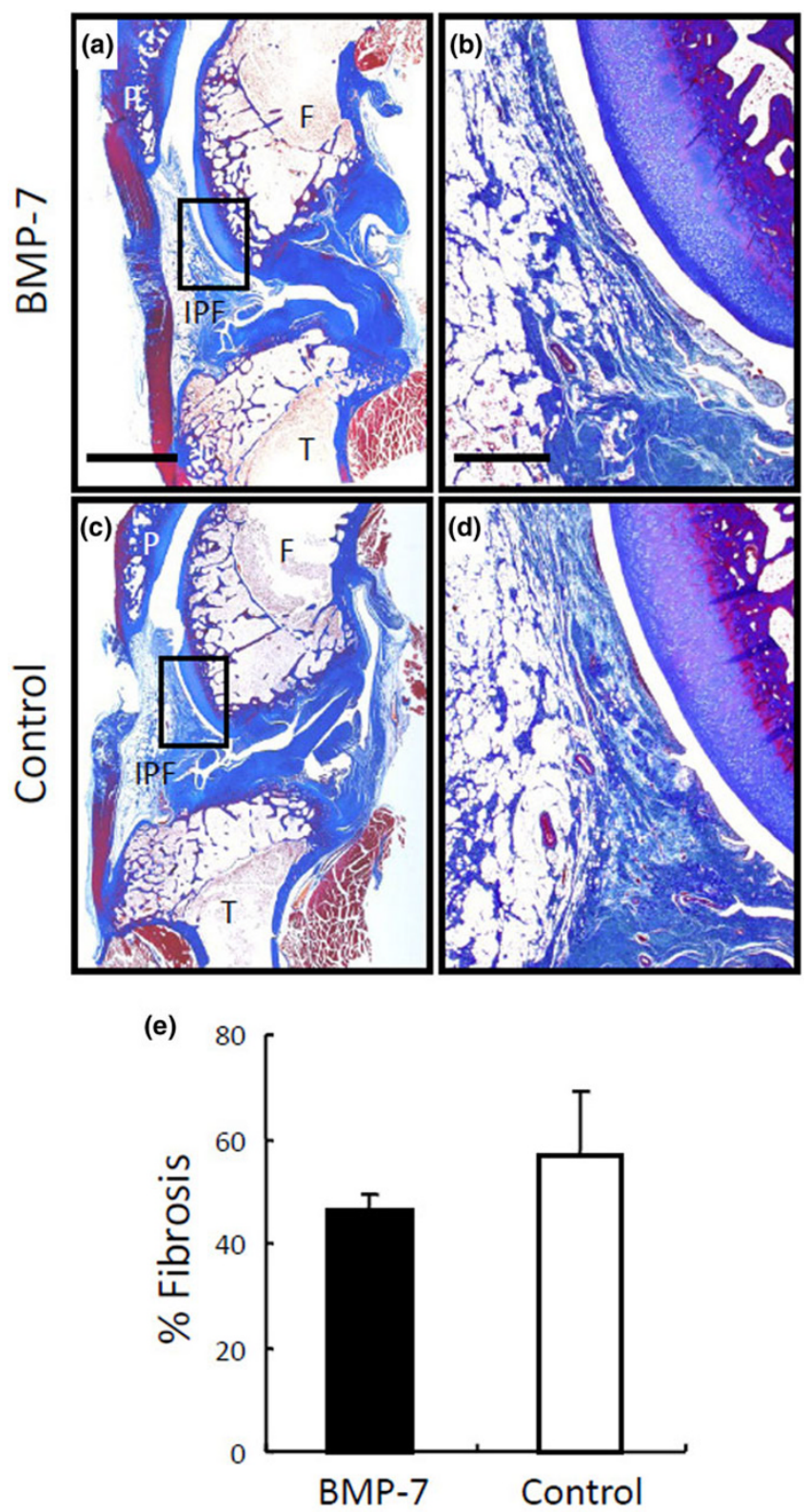

Evaluation of synovial fibrosis. After anterior cruciate ligament transection in both knees, 500 ng BMP-7 in $200 \mu$ PBS was injected weekly into the right knee and the same amount of PBS was injected into the left knee. Whole knee sections stained with Masson's trichrome were evaluated at 12 weeks after ACLT. (a, c) Lower magnified histologies. Bar $=5 \mathrm{~mm}$. (b, d) Higher magnified histologies of infrapatella fat pad. Bar $=1 \mathrm{~mm}$. (e) Quantitation of fibrosis in infrapatellar fat pad. The values are displayed as average \pm standard deviation $(n=3)$. BMP, bone morphogenetic protein; F, femur; IPF, infrapatellar fat pad; P, patella; PBS, phosphate-buffered saline; T, tibia.

would be more attractive. This may also allow the clinician flexibility and options regarding the frequency or duration of the BMP administration, and the potential to adjust the dose delivered into the knee, based on the stage of osteoarthritis. 
Figure 5

(a)

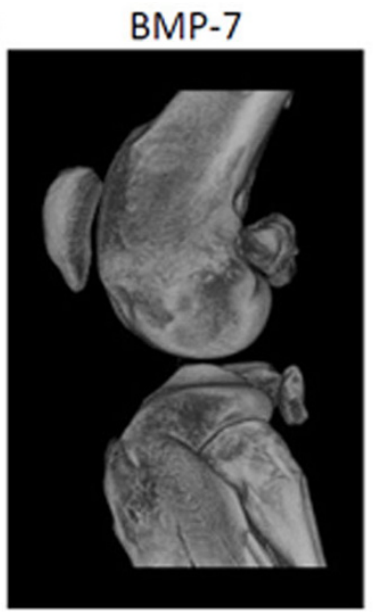

(b)

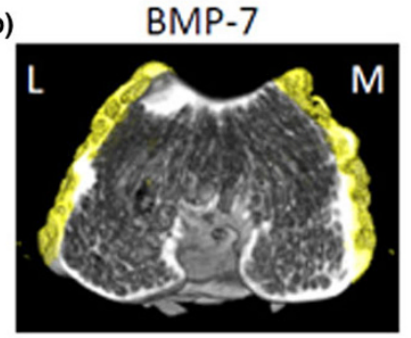

(c)

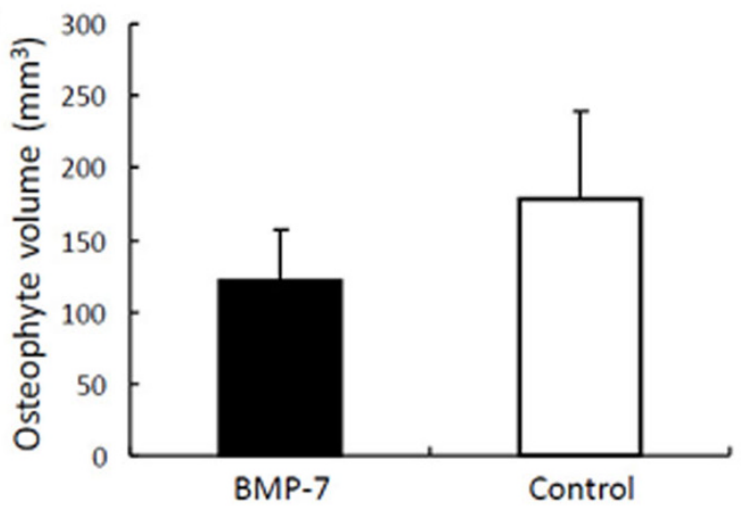

Evaluation of ectopic bone and osteophyte formation. After anterior cruciate ligament transection in both knees, $500 \mathrm{ng}$ BMP-7 in $200 \mu \mathrm{l}$ PBS was injected weekly into the right knee and the same amount of PBS was injected into the left knee. Knee joints were analyzed using a micro CT scanner at 12 weeks. (a) Representative three-dimensional CT images of BMP-7 treated and control knee. Lateral views show no obvious ectopic bone formation in BMP-7 injected side and control side. (b) Representative reconstructed CT images of distal femoral osteophyte. Osteophytes were colored with yellow by image processing. Laterality is shown as lateral (L) and medial (M). (c) Quantitation of osteophyte volume. The values are displayed as average \pm standard deviation $(n=5)$. BMP, bone morphogenetic protein; CT, computed tomography; PBS, phosphate-buffered saline.

In this study, the BMP-7 injections were administered at 7-day intervals. Our pharmacokinetic analysis demonstrated that BMP-7 concentration in the cartilage tissue decreased rapidly within 1 day and then the diminution rate decreased, but a
Figure 6

(a)

Injection

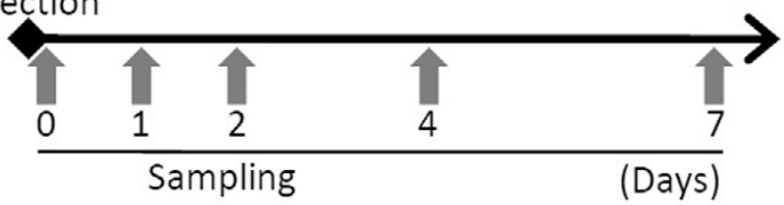

(b)

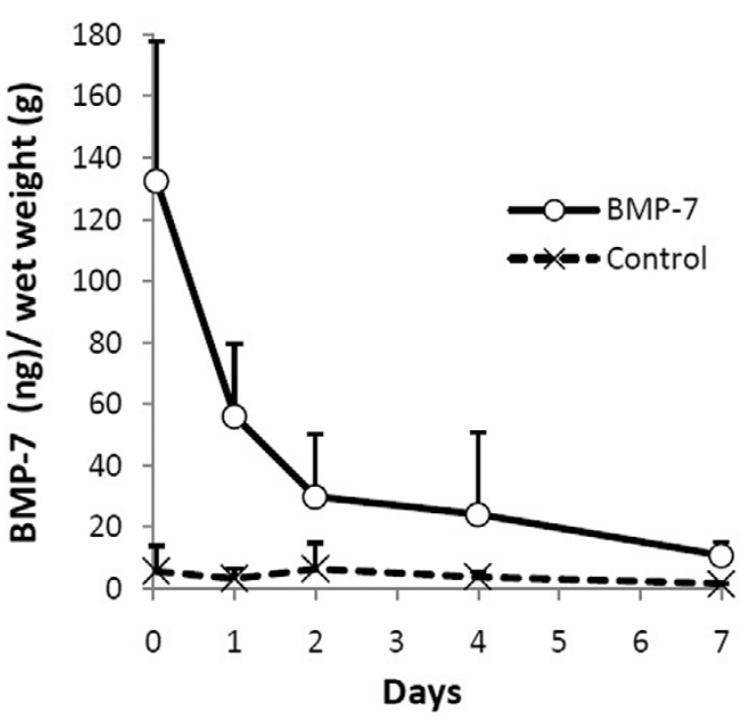

Pharmacokinetic analysis of BMP-7 in cartilage tissue. (a) Outline for the analysis. Cartilage was obtained from knee joints at 1 hour and 1, 2, 4 , and 7 days after intra-articular injection of $500 \mathrm{ng} \mathrm{BMP-7}$ or phosphate-buffered saline. Three joints for each time point were investigated. (b) BMP-7 concentration per cartilage wet weight (ng/g). The values are shown as average \pm standard deviation. BMP, bone morphogenetic protein.

measurable level of BMP-7 was maintained for at least 7 days after the injection. Furthermore, immunohistochemical analysis demonstrated higher BMP-7 expression in chondrocytes from the knee 7 days after the injection. There are three possible mechanisms to explain why the effect of BMP-7 on chondrocytes persisted for more than 7 days after knee injection. First, injected BMP-7 remained in the knee joint during activity for over 7 days. Second, exogenous BMP-7 induced endogenous BMP-7 expression in chondrocytes, and then chondrocytes continued to express endogenous BMP-7 in an autocrine/ paracrine manner. Third, synovial tissue absorbed injected BMP-7, and then synovial cells expressed endogenous BMP7 to enhance endogenous BMP-7 expression in chondrocytes [22,23].

In this rabbit ACLT model, we examined the optimal dose of BMP-7 for the prevention of osteoarthritis progression. First, all knees of rabbits that underwent bilateral ACLT were randomly assigned to one of four treatment groups. Although we demonstrated that both $500 \mathrm{ng}$ and 5,000 ng BMP-7 injections prevented the progression of osteoarthritis, there was no 
significant difference between the two groups. We assume that use of a higher-dose BMP-7 may increase the risk of adverse effects including ectopic cartilage and bone formation, osteophytes, and synovial fibrosis in the knee joint. These doses were much less than those used in other rabbit cartilage studies previously conducted. Sellers and coworkers [16] reported that $5 \mu \mathrm{g}$ BMP-2 promoted healing of full-thickness defects of articular cartilage. In another study employing a similar design [24], $20 \mu \mathrm{g}$ BMP-2 was used with a synthetic biodegradable carrier. The amount of $500 \mathrm{ng}$ BMP- 7 we used for one injection was only $2.5 \%$ to $10 \%$ of that in the previous reports.

The results of our first study indicate considerable individual animal variability in osteoarthritis progression after ACLT. We confirmed that the anterior cruciate ligament was completely transected in every knee when the animals were killed, so the injury was uniform in all animals. Therefore, another mechanism must be responsible for the variability. We suspect that differences in activity level between animals and genetic factors may be responsible.

In our second series of experiments, we wished to address the inter-animal variability observed in the first study and to investigate the effect of BMP-7 on osteoarthritis progression in a stricter manner. Therefore, we tested both treatments (500 ng BMP-7 and PBS alone) in the same animal, with each knee receiving a different treatment. This matched pair analyses demonstrated that both macroscopic and microscopic scores were better in the BMP-7 injected knees than in the contralateral control knees in all rabbits at 12 weeks. This provides further evidence that BMP-7 is effective in preventing OA progression and that the previously observed results were probably not due to the individual variability of rabbits.

The initial pathological change in osteoarthritis is characterized by a depletion of the cartilage extracellular matrix. Intraarticular treatment with BMP-7 may enhance the synthesis of new cartilage matrix, possibly preventing further degeneration. Several in vitro studies indicated that BMP-7 promotes the production of type II collagen and proteoglycans in normal chondrocytes $[1,4]$. Even chondrocytes from osteoarthritic patients have been shown to retain their ability to respond to BMP-7, which also upregulates anabolic gene expression in cartilage $[2,3,5]$.

In consideration of drug efficacy and future clinical use, weekly injections are an attractive option; however, further examination is needed to determine the optimal duration of the BMP-7 injection therapy for the osteoarthritic knee, based on several additional in vivo studies.

\section{Conclusion}

Weekly intra-articular injections of BMP-7 inhibited osteoarthritis progression. BMP-7 concentration and expression in cartilage were still elevated 7 days after BMP-7 injection. No obvious adverse effects resulted from repeated intra-articular injections of BMP-7.

\section{Competing interests}

The authors declare that they have no competing interests.

\section{Authors' contributions}

$\mathrm{MH}$ carried out the animal experiments, analyzed the results, and drafted the manuscript. YJ and TMo participated in the evaluation of the results. TMu designed the initial plan for the study and participated in the evaluation of the results. IS conducted the experiments, participated in the evaluation, and completed the final manuscript. All authors read and approved the final manuscript.

\section{Acknowledgements}

The work was supported by Stryker Biotech.

We thank Kenichi Shinomiya, MD, PhD for continuous support; Ms. Miyoko Ojima for expert help with histology; and Mr. Denis Schrier, Ms.

Nami Migita, and Mr. Kevin Downey for their advice and proofreading.

\section{References}

1. Flechtenmacher J, Huch K, Thonar EJ, Mollenhauer JA, Davies SR, Schmid TM, Puhl W, Sampath TK, Aydelotte MB, Kuettner KE: Recombinant human osteogenic protein 1 is a potent stimulator of the synthesis of cartilage proteoglycans and collagens by human articular chondrocytes. Arthritis Rheum 1996, 39:1896-1904.

2. Fan Z, Chubinskaya S, Rueger DC, Bau B, Haag J, Aigner T: Regulation of anabolic and catabolic gene expression in normal and osteoarthritic adult human articular chondrocytes by osteogenic protein-1. Clin Exp Rheumato/ 2004, 22:103-106.

3. Stove J, Schneider-Wald B, Scharf HP, Schwarz ML: Bone morphogenetic protein 7 (BMP-7) stimulates proteoglycan synthesis in human osteoarthritic chondrocytes in vitro. Biomed Pharmacother 2006, 60:639-643.

4. Nishida Y, Knudson CB, Eger W, Kuettner KE, Knudson W: Osteogenic protein 1 stimulates cells-associated matrix assembly by normal human articular chondrocytes: up-regulation of hyaluronan synthase, CD44, and aggrecan. Arthritis Rheum 2000, 43:206-214.

5. Nishida Y, Knudson CB, Knudson W: Osteogenic Protein-1 inhibits matrix depletion in a hyaluronan hexasaccharideinduced model of osteoarthritis. Osteoarthritis Cartilage 2004, 12:374-382.

6. Chubinskaya S, Kumar B, Merrihew C, Heretis K, Rueger DC Kuettner KE: Age-related changes in cartilage endogenous osteogenic protein-1 (OP-1). Biochim Biophys Acta 2002, 1588:126-134.

7. Badlani N, Inoue A, Healey R, Coutts R, Amiel D: The protective effect of OP-1 on articular cartilage in the development of osteoarthritis. Osteoarthritis Cartilage 2008, 16:600-606.

8. Inoue A, Takahashi KA, Arai $Y$, Tonomura H, Sakao K, Saito M, Fujioka M, Fujiwara $\mathrm{H}$, Tabata $Y$, Kubo T: The therapeutic effects of basic fibroblast growth factor contained in gelatin hydrogel microspheres on experimental osteoarthritis in the rabbit knee. Arthritis Rheum 2006, 54:264-270.

9. Pritzker KP, Gay S, Jimenez SA, Ostergaard K, Pelletier JP, Revell $P A$, Salter D, Berg WB van den: Osteoarthritis cartilage histopathology: grading and staging. Osteoarthritis Cartilage 2006, 14:13-29.

10. Murakami S, Muneta T, Furuya K, Saito I, Miyasaka N, Yamamoto $\mathrm{H}$ : Immunohistologic analysis of synovium in infrapatellar fat pad after anterior cruciate ligament injury. Am J Sports Med 1995, 23:763-768. 
11. Zhang B, Muneta T, Yagishita K, Sekiya I: Substance P immunoreactive fibers of synovial tissue in patients with anterior cruciate ligament injury. Knee Surg Sports Traumatol Arthrosc 2006, 14:404-410.

12. Sah RL, Yang AS, Chen AC, Hant JJ, Halili RB, Yoshioka M, Amiel $\mathrm{D}$, Coutts RD: Physical properties of rabbit articular cartilage after transection of the anterior cruciate ligament. J Orthop Res 1997, 15:197-203.

13. Setton LA, Elliott DM, Mow VC: Altered mechanics of cartilage with osteoarthritis: human osteoarthritis and an experimental model of joint degeneration. Osteoarthritis Cartilage 1999, 7:2-14.

14. McDevitt $\mathrm{CA}$, Muir $\mathrm{H}$ : Biochemical changes in the cartilage of the knee in experimental and natural osteoarthritis in the dog. $J$ Bone Joint Surg Br 1976, 58:94-101.

15. Yoshioka M, Coutts RD, Amiel D, Hacker SA: Characterization of a model of osteoarthritis in the rabbit knee. Osteoarthritis Cartilage 1996, 4:87-98.

16. Sellers RS, Zhang R, Glasson SS, Kim HD, Peluso D, D'Augusta DA, Beckwith K, Morris EA: Repair of articular cartilage defects one year after treatment with recombinant human bone morphogenetic protein-2 (rhBMP-2). J Bone Joint Surg Am 2000, 82:151-160.

17. Grgic M, Jelic M, Basic V, Basic N, Pecina M, Vukicevic S: Regeneration of articular cartilage defects in rabbits by osteogenic protein-1 (bone morphogenetic protein-7). Acta Med Croatica 1997, 51:23-27.

18. Jelic M, Pecina M, Haspl M, Kos J, Taylor K, Maticic D, McCartney J, Yin S, Rueger D, Vukicevic S: Regeneration of articular cartilage chondral defects by osteogenic protein-1 (bone morphogenetic protein-7) in sheep. Growth Factors 2001, 19:101-113.

19. Louwerse RT, Heyligers IC, Klein-Nulend J, Sugihara S, van Kampen GP, Semeins CM, Goei SW, de Koning MH, Wuisman PI, Burger $\mathrm{EH}$ : Use of recombinant human osteogenic protein-1 for the repair of subchondral defects in articular cartilage in goats. J Biomed Mater Res 2000, 49:506-516.

20. Cook SD, Patron LP, Salkeld SL, Rueger DC: Repair of articular cartilage defects with osteogenic protein-1 (BMP-7) in dogs. $J$ Bone Joint Surg Am 2003, 85-A(suppl 3):116-123.

21. Mattioli-Belmonte M, Gigante A, Muzzarelli RA, Politano R, De Benedittis A, Specchia N, Buffa A, Biagini G, Greco F: N,N-dicarboxymethyl chitosan as delivery agent for bone morphogenetic protein in the repair of articular cartilage. Med Biol Eng Comput 1999, 37:130-134.

22. Luyten FP, Yu YM, Yanagishita M, Vukicevic S, Hammonds RG, Reddi AH: Natural bovine osteogenin and recombinant human bone morphogenetic protein-2B are equipotent in the maintenance of proteoglycans in bovine articular cartilage explant cultures. J Biol Chem 1992, 267:3691-3695.

23. Koepp HE, Sampath KT, Kuettner KE, Homandberg GA: Osteogenic protein-1 (OP-1) blocks cartilage damage caused by fibronectin fragments and promotes repair by enhancing proteoglycan synthesis. Inflamm Res 1999, 48:199-204.

24. Tamai N, Myoui A, Hirao M, Kaito T, Ochi T, Tanaka J, Takaoka K, Yoshikawa $\mathrm{H}$ : A new biotechnology for articular cartilage repair: subchondral implantation of a composite of interconnected porous hydroxyapatite, synthetic polymer (PLA-PEG), and bone morphogenetic protein-2 (rhBMP-2). Osteoarthritis Cartilage 2005, 13:405-417. 